\title{
Advances in the DNA methylation hydroxylase TET1
}

\author{
Wenzheng Liu, Guanhua Wu, Fei Xiong and Yongjun Chen ${ }^{*}$
}

\begin{abstract}
Background: The ten-eleven translocation 1 (TET1) protein is a 5-methylcytosine hydroxylase that belongs to the TET protein family of human a-ketoglutarate oxygenases. TET1 recognizes and binds to regions of high genomic 5'CpG-3' dinucleotide density, such as CpG islands, initiates the DNA demethylation program, and maintains DNA methylation and demethylation balance to maintain genomic methylation homeostasis and achieve epigenetic regulation. This article reviews the recent research progress of TET1 in the mechanism of demethylation, stem cells and immunity, various malignant tumours and other clinical diseases.

Conclusion: TET1 acts as a key factor mediating demethylation, the mechanism of which still remains to be investigated in detail. TET1 is also critical in maintaining the differentiation pluripotency of embryonic stem cells and plays anti- or oncogenic roles in combination with different signalling pathways in different tumours. In certain tumours, its role is still controversial. In addition, the noncatalytic activity of TET1 has gradually attracted attention and has become a new direction of research in recent years.

Keywords: Ten-eleven translocation 1, DNA demethylation, Stem cells, Immunity, Clinical disease, Malignant tumours, Leukaemia, Breast cancer, Lung cancer, Hepatocellular carcinoma, Cholangiocarcinoma, colon cancer, Pancreatic cancer, Prostate cancer, Cervical cancer, Metabolism, Neurological system
\end{abstract}

\section{Background}

DNA methylation, an important epigenetic modification, has a profound impact on genome stability, transcription and development. This term specifically refers to the conversion of a methyl group onto the $\mathrm{C} 5$ position of 5'-CpG-3' dinucleotides to form 5-methylcytosine $(5 \mathrm{mC})$, which is catalysed by DNA methyltransferase (DNMT) with S-adenosyl methionine (SAM) as the active methyl donor. DNA methylation does not change the sequence of DNA bases and has reversible biological properties, namely, the reduction of $5 \mathrm{mC}$ to 5 -cytosine (5C) after DNA methylation is catalysed by "demethylase". Cells rely on DNA methyltransferases and demethylases together to maintain genomic methylation

\footnotetext{
* Correspondence: Yjchen@tjh.tjmu.edu.cn

Department of Biliary and Pancreatic Surgery, Tongji Hospital, Tongji Medical College, Huazhong University of Science and Technology, 1095 Jiefang Avenue, Wuhan 430030, Hubei, China
}

homeostasis. Imbalances in genomic methylation homeostasis contribute to various diseases, including cancer.

The mechanism of DNA demethylation is considerably complicated; it has been classified as active demethylation and passive demethylation and involves various 5methylcytosine catalases. Active DNA demethylation refers to an enzymatic process that removes or modifies the methyl group from $5 \mathrm{mC}$. In contrast, passive DNA demethylation refers to loss of $5 \mathrm{~m} 0 \mathrm{C}$ during successive rounds of replication in the absence of functional DNA methylation maintenance machinery. The ten-eleven translocation (TET) protein family is a key catalytic protein that mediates DNA demethylation and plays an important role in both active and passive demethylation processes. The human TET protein family has three members, TET1, TET2 and TET3, of which TET1, a $5 \mathrm{mC}$ hydroxylase, is closely associated with

(c) The Author(s). 2021 Open Access This article is licensed under a Creative Commons Attribution 4.0 International License, which permits use, sharing, adaptation, distribution and reproduction in any medium or format, as long as you give appropriate credit to the original author(s) and the source, provide a link to the Creative Commons licence, and indicate if changes were made. The images or other third party material in this article are included in the article's Creative Commons licence, unless indicated otherwise in a credit line to the material. If material is not included in the article's Creative Commons licence and your intended use is not permitted by statutory regulation or exceeds the permitted use, you will need to obtain permission directly from the copyright holder. To view a copy of this licence, visit http://creativecommons.org/licenses/by/4.0/. The Creative Commons Public Domain Dedication waiver (http://creativecommons.org/publicdomain/zero/1.0/) applies to the data made available in this article, unless otherwise stated in a credit line to the data. 
tumorigenesis and progression through its abnormal expression and/or function.

\section{Basic characteristics of TET1}

TET1 was the first to be defined in the human TET protein family and was identified as a fusion protein by Ono $\mathrm{R}$ et al. in a study of a special patient with acute leukaemia [1]. TET1 was named the ten-eleven translocation (TET) protein based on the presence of t $(10 ; 11)$ (q 22; q 23) ectopically in this patient. Subsequently, Tahiliani et al. [2] discovered the TET family of proteins in mammals in 2009 and later was the first to identify that TET1 could catalyse the hydroxylation of $5 \mathrm{mC}$ in vitro. TET1 has a cysteine-rich domain $(\mathrm{CD})$ at the $\mathrm{C}$-terminus and a Cys-Xaa-Xaa-Cys (CXXC) domain at the $\mathrm{N}$-terminus. Multiple $\beta$-chains form a double-stranded b-helix (DSBH) with 2OG$\mathrm{Fe}^{2+}$-dependent oxidase characteristics together with a CD domain to form the catalytic domain of TET1 [3-5], allowing TET1 to recognize and bind unmodified, 5mC-modified and 5-hydroxymethylcytosine (5hmC)-modified CpG-DNA [6, 7].

\section{TET1 initiates the process of DNA demethylation}

In the mammalian genome, $5 \mathrm{mC}$ is mainly distributed in CpG dinucleotides, and approximately $70-80 \%$ of CpGs are methylated [8]. TET1 is an active $5 \mathrm{mC}$ hydroxylase that hydroxylates $5 \mathrm{mC}$ to $5 \mathrm{hmC}$ in vivo and in vitro, thus initiating the DNA demethylation process [9]. Three main mechanisms of TET1-mediated demethylation have been described.

\section{TET1 protein prevents DNA from maintenance methylation}

In DNA replication, hemimethylated $(\mathrm{M} / \mathrm{C})$ and hemihydroxymethylated $(\mathrm{H} / \mathrm{C}) \mathrm{CpG}$ dinucleotides are produced transiently, where only the parental strand is modified while the daughter strand remains an unmodified cytosine. DNA maintenance methylation is mediated by DNA methyltransferase 1 (DNMT1) and ubiquitin-like with PHD and ring finger domains 1 (UHRF1). UHRF1 binds to the hemimethylated CG site through the SAD/ SRA structural domain and recruits DNMT1, which interacts with the CG site to maintain the DNA methylation pattern during cytokinesis [10]. TET1 catalyses initial DNA strand methylation site hydroxylation to $5 \mathrm{hmC}$. In vitro experiments show that DNMT1 is 50fold more active intrinsically against $\mathrm{M} / \mathrm{C}$ substrates than against $\mathrm{H} / \mathrm{C}$ substrates and that UHRF1 binds 10fold less well to $\mathrm{H} / \mathrm{C}$ substrates than to $\mathrm{M} / \mathrm{C}$ substrates $[11,12]$. The above studies show that in vivo, TET1 prevents UHRF1 and DNMT1 from binding to the daughter strand by catalysing the parental strand $5 \mathrm{mC}$ to $5 \mathrm{hmC}$, thereby hindering the maintenance of DNA methylation and thus passively eliminating $5 \mathrm{mC}$ by replication. However, Kubosaki A [13] et al. stably transferred hydroxymethylated plasmids and methylated plasmids into human cells separately and found that both were maintained to the same extent for methylation during cell replication and division, suggesting that $5 \mathrm{hmC}$ does not completely hinder the maintenance of methylation in cells and that the molecular mechanism for the presence of hemihydroxymethylated $\mathrm{CpG}$ recognition has not been explored (Fig. 1).

\section{TET1-mediated active demethylation in DNA repair}

In contrast to the extent of passive dilution of DNA methylation dependent on DNA replication, active demethylation is not dependent on DNA replication and is closely related to the oxidative activity of TET1 on $5 \mathrm{mC}$ and base excision repair (BER). TET1 first hydroxylates $5 \mathrm{mC}$ to $5 \mathrm{hmC}$ as a demethylation intermediate, and the next conversion of $5 \mathrm{hmC}$ may involve two aspects.

On the one hand, studies on DNA demethylation point to a mechanism involving TET and thymidineDNA glycosylase (TDG) [7]. Current studies suggest that this pathway is formed by gradually catalysed oxidation of $5 \mathrm{mC}$ by TET 1 to form the TDG-dependent BER substrates 5-formylcytosine (5fC) and 5-carboxylcytosine $(5 \mathrm{caC})$, followed by the replacement of $5 \mathrm{mC}$ with $\mathrm{C}$ to restore the unmethylated DNA sequence [6]. TDG is a protein which mediates DNA repair, that recognizes various cytosine and $5 \mathrm{mC}$ base derivatives in DNA [14]. TDG has long been a focus of biochemical research, as T.G or hmU.G mismatches are known TDG substrates, and TDG can excise base T and replace unmodified $C$ during genomic T.G mismatches [15]. This biochemical pathway is supported by TET and TDG knockout mice and embryonic stem cells [16]. The involvement of the BER system implies the generation of apurinic/apyrimidinic (AP) sites [14]. The AP site is first cleaved by apurinic aprimidinic endonuclease/Redox factor1(APE1/Ref-1), an enzyme that produces DNA singlestrand breaks followed by DNA gap filling and ligation by activating poly ADP-ribose polymerase $1, \mathrm{X}$-ray repair cross complementary protein 1 (XRCC1), DNA ligase 3 (LIG3), and DNA polymerase b (POLb) [16]. Thus, TET1 and TDG initiate DNA demethylation by oxidizing and excising $5 \mathrm{mC}$ from DNA.

On the other hand, Guo,J et al. [17] revealed in virustransfected human cells and mouse brains that $5 \mathrm{hmC}$ can be deaminated by activation-induced deaminase (AID) and apolipo protein B mRNA-editing enzymecatalysed polypeptide-like (APOBEC) protein. APOBEC catalyses deamination of 5-hydroxymethyluracil ( $5 \mathrm{hmU})$, which is then recognized and excised by TDG [18]. Then, the site is converted to cytosine via the BER pathway, which achieves DNA demethylation. These 


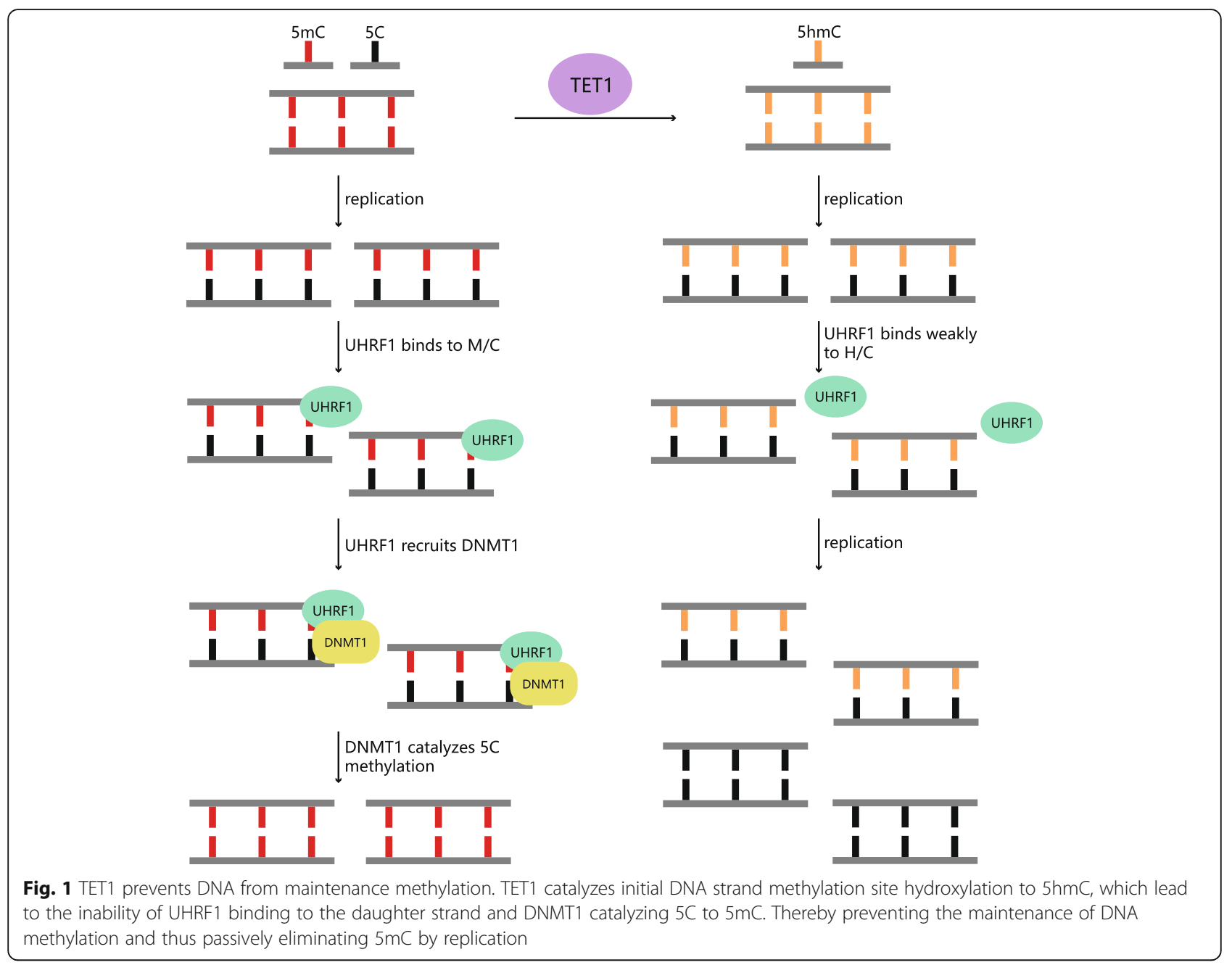

deamination-mediated demethylation pathways may also involve the DNA damage response protein growth arrest and DNA damage inducible 45 (GADD45), and methylCpG-binding domain 4 (MBD4) may even be used as a glycosylase alternative for excision of T-G mismatches [19-21]. In human cells cultured in vitro, AID/APOBEC deaminase specifically promoted $5 \mathrm{hmC}$ demethylation but had no significant effect on $5 \mathrm{mC}$. In the adult mouse brain, AID contributed to endogenous $5 \mathrm{hmC}$ removal. In addition, TET1 and AID facilitate region-specific demethylation of neurons in the dentate region [10]. This mechanism has been found in stonefish [20], neurons [17], human tumour cells [22] and primordial germ cells [23]. However, the mechanism remains controversial, with some studies showing that AID does not interact with double-stranded DNA (dsDNA) and that the $5 \mathrm{mC}$ responsiveness to AID deamination in vitro is much lower than that of nonmethylated cytosine [24]. Christopher S. Nabel et al. [25] found that the activity of purified AID/APOBEC at $5 \mathrm{mC}$ was greatly reduced relative to its conventional substrate cytosine, and all AID/
APOBEC family members preferentially interact with unmodified cytosine. Furthermore, after AID/APOBEC overexpression, no significant expression of the deamination product of $5 \mathrm{hmC}$ was detected in genomic DNA, whereas intermediates in the iterative oxidative pathway were readily found in genomic DNA and their formation was not reduced by deaminase overexpression. After a series of reactivity studies of the intermediates, it was found that their spatial volume became increasingly unfavourable for deamination, and therefore this study concluded that the spatial requirement for cytosine deamination is an intrinsic obstacle for deaminase to perform its DNA demethylation function.

To summarize, deaminases play a limited function in DNA demethylation relative to the oxidation-mediated pathway (Fig. 2).

\section{TET-mediated decarboxylation of $5 \mathrm{caC}$}

Direct decarboxylation of $5 \mathrm{caC}$ to achieve demethylation has a high energy barrier, but Schiesser $\mathrm{S}$ et al. [26] studied $5 \mathrm{caC}$ isotope labelling and demonstrated the 


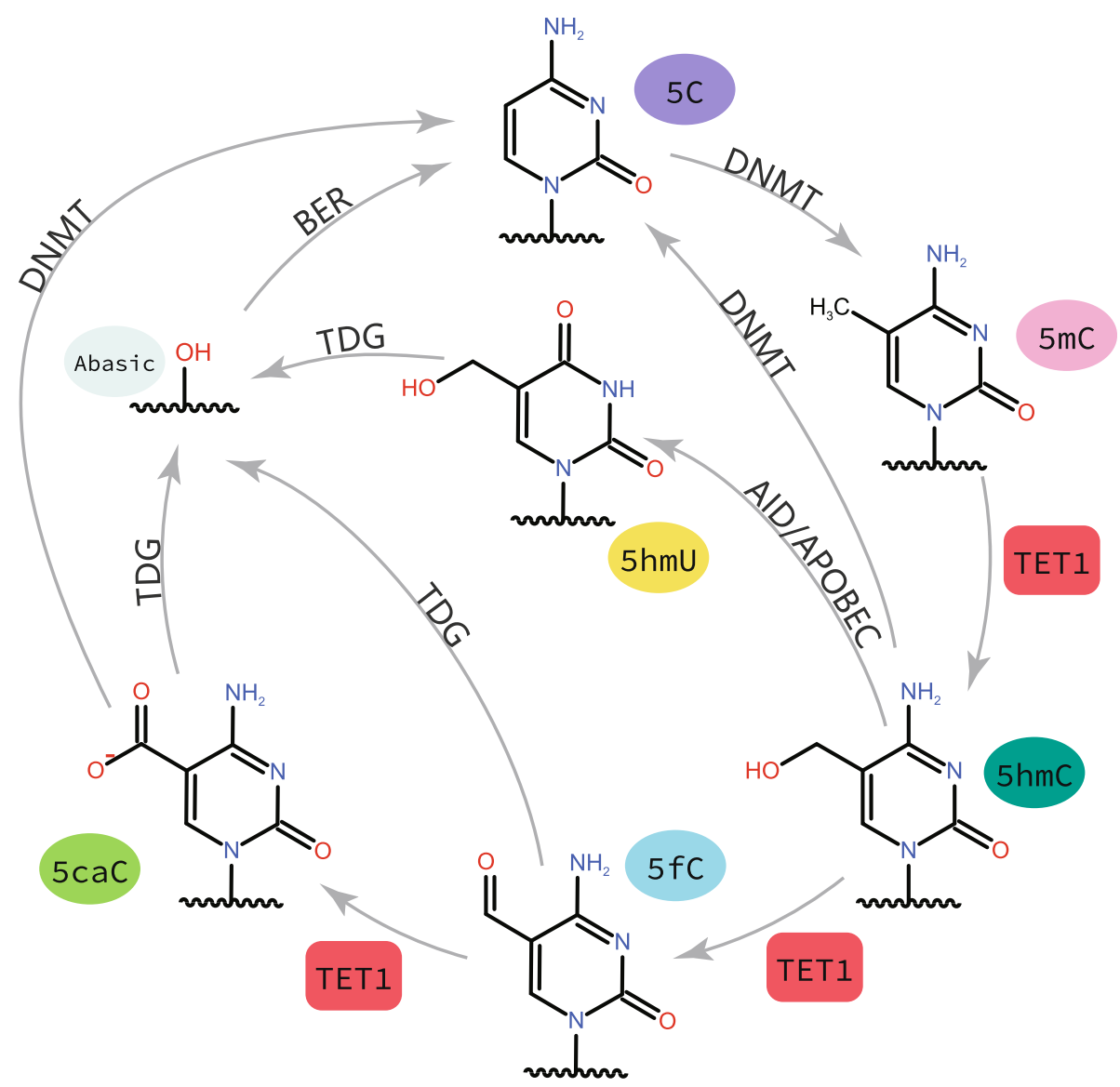

Fig. 2 TET1-mediated active DNA demethylation. A complete model of TET1-mediated active DNA demethylation is shown. $5 \mathrm{mC}$ is formed by DNMT. TET1 gradually catalyze the oxidation of $5 \mathrm{mC}$ to form $5 \mathrm{hmC}, 5 \mathrm{fC}$ and $5 \mathrm{caC}$. Among which $5 \mathrm{hmC}$ and $5 \mathrm{caC}$ can be eliminate the hydroxymethyl group and the carboxyl group to generate unmodified cytosine directly by DNMT, and $5 \mathrm{hmC}$ can also be catalyzed deamination by AID/APOBEC to $5 \mathrm{hmU} .5 \mathrm{hmU}$ along with $5 \mathrm{fC}$ and $5 \mathrm{caC}$ formed in the previous steps can be recognized and excised by TDG to generate an abasic site as part of the base excision repair (BER) process that regenerates unmodified C

feasibility of direct removal of oxidized amino acids; that is, $5 \mathrm{caC}$ can be directly decarboxylated and reduced to unmodified $\mathrm{C}$ in the presence of lysis products from embryonic stem cells. Oligonucleotides containing $5 \mathrm{caC}$ are synthesized and repaired in the presence of lysis products from embryonic stem cells, where the pyrimidine ring of $5 \mathrm{caC}$ is labelled by ${ }^{15} \mathrm{~N}$. A small amount of ${ }^{15} \mathrm{~N}_{2}$ deoxycytidine was detected in the repair and disassembly of the oligonucleotide, indicating that $5 \mathrm{caC}$ can be directly decarboxylated to $\mathrm{C}$ in the absence of BER. Moreover, this pathway has a precedent for heterologous acid decarboxylase.

Zita Liutkeviciute et al. [27] demonstrated that both bacterial and mammalian DNMTs may catalyse the direct decarboxylation of $5 \mathrm{caC}$ to generate unmodified cytosine in DNA in vitro and can eliminate the hydroxymethyl group of $5 \mathrm{hmC}$, directly converting $5 \mathrm{hmC}$ to unmodified $\mathrm{C}$, but are inert to $5 \mathrm{fC}$. Under simulated enzyme-catalysed conditions (high concentrations of exogenous thiols and imidazole, $\mathrm{pH}=5.0$ ), the $\mathrm{C}-\mathrm{C}$ bond breakage of all three oxidized cytosines occurs in the following rate order: $5 \mathrm{CaC}>5 \mathrm{fC}>5 \mathrm{hmC}$. Reducing conditions favour the onset of DNMT3a methyl transfer, whereas oxidizing conditions favour DNMT-mediated dehydroxymethylation. The atypical $\mathrm{C}-\mathrm{C}$ bond cleavage reaction provides the possibility of direct "reversal" of $5 \mathrm{caC}$ to unmodified C. However, the direct decarboxylation of $5 \mathrm{caC}$ by $5 \mathrm{caC}$ decarboxylase in vivo has not been determined, and further studies are needed (Fig. 2).

\section{TET1 with stem cells and immunity TET1 with stem cells}

TET1 and 5hmC have essential roles in maintaining the pluripotent state of embryonic stem cells (ESCs). In 2010 Shinsuke Ito et al. [3] studied mouse embryonic stem cells and found that TET1 has an important role in mouse embryonic stem (ES) cell maintenance by maintaining the expression of Nanog in ES cells. 
Downregulation of Nanog via TET1 knockdown correlates with methylation of the Nanog promoter, supporting a role for TET1 in regulating DNA methylation status. Furthermore, knockdown of TET1 in preimplantation embryos results in a bias towards trophectoderm differentiation. Their studies not only uncovered the enzymatic activity of TET proteins but also demonstrated a role for TET1 in ES cell maintenance and inner cell mass cell specification.

Subsequently, numerous studies have shown that TET1 binds primarily to the transcription start site of the CpG-rich promoter of the embryonic stem cell genome and regulates the level of DNA methylation in the promoter of the CpG-rich region. The competitive balance between the TET1 protein and DNMT at transcription start sites and other genomic loci promotes rapid changes with methylation status, activating or silencing transcription in a cell line and site-specific manner [28]. TET1 uses a two-pronged approach to maintain the selfrenewal capacity and pluripotency potential of embryonic stem cells by "silencing" differentiation genes on the one hand and "activating" pluripotency genes on the other. The "silencing" of differentiation genes by TET1 is closely related to the transcriptional regulation of polycomb repressive complex 2 (PRC2). PRC2 recruits DNMT3a and DNMT3b to the promoters of genes involved in germ cell differentiation and mediates the enrichment of tri-methylation at lysine 27 of histone $\mathrm{H} 3$ (H3K27me3) to initiate de novo methylation [29, 30]. In the CpG-enriched region, DNA methylation prevents the binding of embryonic ectoderm development/enhancer of zeste 2/suppressor of zeste 12 (EED/EZH2/ SUZ12, the core of the PRC2 subunit) to specific promoter regions of chromatin, whereas TET1 can preferentially bind to the $\mathrm{CpG}$ region, maintaining a hypermethylated state of the human embryonic stem cell promoter and ensuring that strong spectrum-specific transcription is maintained after differentiation by preventing $a b$ initio methylation of the human embryonic stem cell promoter [31-33].

Notably, promoter DNA methylation does not immediately lead to transcriptional changes in human embryonic stem cells but can still impair their differentiation [3]. TET1 regulates many genes that define ectodermal and extraembryonic ectodermal differentiation programs. In ectodermal cells, TET1 demethylates gene promoters and maintains telomere stability through hydroxymethylation [34]. A series of studies have shown that knockdown of TET1 alone or co-knockdown of TET1 and TET2 in mouse embryonic stem cells leads to difficulties in maintaining embryonic stem cell pluripotency and skewed differentiation towards ectoderm and primitive endoderm [34]. TET1 maintains the "activation" of pluripotent genes by demethylation of gene promoters and represses most epistatic target genes independent of methylation changes by regulating the expression of the gene encoding the transcriptional repressor Jumonji domain-containing protein 8 (JMJD8) [34]. In primordial germ cell meiosis, DNA methylation, polycomb repressive complex 1 (PRC1) and TET1mediated DNA demethylation activate a key set of germ cell reprogramming response genes involved in gamete production and meiosis [35].

Surprisingly, independent of methylation changes TET1 can also repress most ectodermal target genes, in part by regulating the gene encoding the transcriptional repressor JMJD8. In the absence of TET1, dysregulation of gene expression results in embryonic defects that are partially penetrant in inbred strains but completely lethal in noninbred mice [34].

In bone marrow mesenchymal stem cells (BMMSCs), TET1 plays a key role in maintaining BMMSC and bone homeostasis by controlling exosome and miRNA release through P2X purinoceptor 7 (P2RX7) demethylation, and could be a target for the development of new therapies for osteopenia [36].

Overall, the present study highlights the interplay between the catalytic and noncatalytic activities of TET1, which is essential for normal development.

\section{TET1 with immunity}

There is growing evidence of a link between DNA methylation and antitumour immunity. Hao-Xiang Wu et al. [37] collected and integrated clinical cohorts from published studies with annotated response and survival data as well as matched mutation data. The predictive function of specific mutated genes was first tested in a discovery cohort, later validated in a validation cohort, and further investigated in The Cancer Genome Atlas (TCGA) dataset to examine the relationship between specific mutated genes and tumour immunogenicity and antitumour immunity. They found that among 21 key genes involved in regulating DNA methylation, the TET1 mutation (TET1-MUT) was enriched in patients who responded to immune checkpoint inhibitor (ICI) therapy in the discovery cohort $(p=0.003)$. In the discovery cohort $(n=519)$, patients with TET1-MUT and TET1-wild-type (TET1-WT) were enriched in objective response rate (ORR, $60.9 \%$ versus $22.8 \%, P<0.001$ ), durable clinical benefit (DCB, 71.4\% versus $31.6 \%, P<0.001$ ) and progression-free survival (PFS, hazard ratio $=0.46$ [95\% confidence interval, 0.25 to 0.82 ], $P=0.008$ ), which were significantly different. In the validation cohort $(n=$ 1395), there was a significant overall survival (OS) advantage for TET1-MUT patients compared to TET1WT patients (hazard ratio $=0.47$ [95\% confidence interval, 0.25 to 0.88 ], $P=0.019$ ), which, importantly, was not associated with tumour mutation burden or high 
microsatellite instability; nor was it attributable to the TET1-MUT prognostic impact $(P>0.05$ for both nonICI treatment cohorts). In the TCGA dataset, TET1MUT was strongly associated with higher tumour mutational burden and neoantigen load, as well as inflammatory patterns, immune features and immune-related gene expression in tumour-infiltrating $\mathrm{T}$ lymphocytes. In patients treated with ICI, TET1-MUT was strongly associated with higher ORR, better DCB, longer PFS and improved OS, suggesting that TET1-MUT is a novel predictive biomarker for immune checkpoint blockade against multiple cancer types.

Regulatory T (Treg) cells are essential for maintaining immune homeostasis, and TET1 and TET2 catalyse the conversion of $5 \mathrm{mC}$ to $5 \mathrm{hmC}$ in forkhead box P3 (FOXP3) to establish Treg cell-specific hypomethylation patterns and stable FOXP3 expression. Thus, TET1 and TET2 deficiency leads to FOXP3 hypermethylation, impaired Treg cell differentiation and function, and autoimmune diseases [38].

\section{TET1 in clinical disease}

TET1 plays different roles in different diseases and subtypes, some of which are still controversial (Table 1).

\section{TET1 in leukaemia}

TET1 was initially identified as a fusion protein in a specific case of acute myeloid leukaemia (AML) and was therefore presumed to be associated with leukaemogenesis. Subsequently, some AML patients were found to have an MLL-TET1 fusion gene, including the carboxyl terminus of TET1 (containing the 2-OG-Fe2+ oxidase structural domain) and the amino terminus of mixed lineage leukaemia (MLL) (containing the CXXC structural domain) [39], playing an important oncogenic role by activating homeobox gene expression.

In 2020 Tingjuan Zhang et al. [40] identified that TET1 expression was significantly reduced in AML patients but was highly expressed in T-cell acute lymphoblastic leukaemia (T-ALL) and essential for its development. In 2021, Shiva Bamezai et al. [41] reported that TET1 is highly expressed in T-ALL and is crucial for human T-ALL cell growth in vivo. Knockout of TET1 in mice and knockdown in human T cells did not perturb normal $\mathrm{T}$-cell proliferation, indicating that TET1 expression is dispensable for normal T-cell growth. The promotion of leukaemic growth by TET1 was dependent on its catalytic property to maintain global 5-hydroxymethylcytosine $(5 \mathrm{hmC})$ levels, thereby regulating the cell cycle, DNA repair genes, and T-ALL associated oncogenes. Furthermore, overexpression of the TET1 catalytic domain was sufficient to augment global $5 \mathrm{hmC}$ levels and leukemic growth of T-ALL cells in vivo. Their study demonstrated that PARP enzymes, which are highly expressed in T-ALL patients, participate in establishing H3K4me3 marks at the TET1 promoter and that PARP1 interacts with the TET1 protein. Importantly, the growth-related role of TET1 in T-ALL could be antagonized by the clinically approved PARP inhibitor olaparib, which abrogated TET1 expression, induced loss of $5 \mathrm{hmC}$, and antagonized leukaemic growth of T-ALL cells, opening a therapeutic avenue for this disease.

The TET protein family often displays opposite expression trends in different types of haematological diseases $[40,42]$. TET2 mutations are frequent in patients with AML, myelodysplastic syndromes (MDS) and chronic myelocytic leukaemia (CML), while TET1 and

Table 1 The expression level of TET1 in different malignant diseases and subtypes

\begin{tabular}{|c|c|c|}
\hline Malignant Diseases & Subtypes & Expression Level of TET1 \\
\hline \multirow[t]{2}{*}{ Leukemia } & AML & Low \\
\hline & T-ALL & High \\
\hline \multirow[t]{3}{*}{ Breast Cancer } & HER2 & Unchanged \\
\hline & HRBC & Low \\
\hline & Triple Negative & High \\
\hline \multirow[t]{2}{*}{ Lung Cancer } & Adenocarcinoma & High \\
\hline & Squamous Cell Carcinoma & High \\
\hline Hepatocellular Carcinoma & - & Low \\
\hline Cholangiocarcinoma & Intrahepatic Cholangiocarcinoma & Low \\
\hline Colon Cancer & - & Low \\
\hline Pancreatic Cancer & - & Low \\
\hline Prostate Cancer & - & Low \\
\hline Cervical Cancer & - & High \\
\hline
\end{tabular}


TET3 mutations are rare [42, 43]. The mechanisms of which have yet to be studied in depth.

\section{TET1 in solid tumours}

The global decrease in $5 \mathrm{hmC}$ with downregulation of TET and/or functional alterations in TET was once considered a hallmark of cancer, and this dysregulation of DNA methylation levels was found in various solid tumours, such as breast, lung, liver, colon, pancreatic and prostate cancers [44-47]. However, the latest studies have raised the possibility that TET1 can be both an oncogene and tumour suppressor gene.

\section{TET1 in breast cancer}

High or low DNA methylation levels are found in breast cancer, but factors regulating the balance of methylation and demethylation remain unclear. However, that the degree of DNA demethylation in breast cancer is closely related to the growth, invasion, and metastasis of breast cancer cells.

In 2012, Chih-Hung Hsu et al. [44] found that TET1 was suppressed in breast cancer tissues. The depletion of TET1 in xenograft models promoted breast cancer cell invasion and growth and induced breast cancer cell metastasis. Overexpression of TET1 inhibits tumour cell invasion and xenograft tumour formation by activating tissue inhibitors of metalloproteinases. In 2018, Naifei Chen et al. [48] found that an exonic circular RNA (FLI1 exonic circular RNA, FECR1) regulates the balance of methylation and demethylation regulating breast cancer growth and promoting breast cancer metastasis by recruiting TET1 together with transDNMT1.

Triple-negative breast cancer (TNBC), a subtype that does not overexpress estrogen receptor, progesterone receptor or human epidermal growth factor receptor 2 (HER2) gene $\left(\mathrm{ER}^{-} / \mathrm{PR}^{-} / \mathrm{HER} 2^{-}\right)$, is a minimally methylated cancer. In 2018, Charly Ryan Good et al. [49] compared of 100 TNBC patients and 732 hormone receptor positive (HRBC) patients with 105 normal subjects. They showed that TET1 expression was significantly increased in TNBC compared to normal breast. Through bioinformatic analyses in both breast and ovarian cancer cell line panels, they uncovered an intricate network connecting TET1 to hypomethylation and activation of cancerspecific oncogenic pathways, including phosphatidylinositol 3-kinase (PI3K), epidermal growth factor receptor (EGFR), and platelet derived growth factor (PDGF). TET1 expression correlated with sensitivity to drugs targeting the PI3K-mTOR pathway, and CRISPRmediated deletion of TET1 in two independent TNBC cell lines resulted in reduced expression of PI3K pathway genes, upregulation of immune response genes, and substantially reduced cellular proliferation, suggesting dependence of oncogenic pathways on TET1 overexpression. Their work establishes TET1 as a potential oncogene that contributes to aberrant hypomethylation in cancer and suggests that TET1 could serve as a druggable target for therapeutic intervention.

In 2019 Yong Yu et al. [50] confirmed that in TNBC cells, EZH2 inhibited TET1 expression and subsequently inhibited the antitumour p53 signalling pathway, and patients with high EZH2 expression and low TET1 expression had the shortest survival. Targeted suppression of $\mathrm{EZH} 2$ in TNBC cells in vitro using the specific inhibitor GSK343 or shRNA can induce cell cycle arrest and cellular senescence by increasing TET1 expression and p53 pathway activation. This study opens a new avenue for TNBC treatment by targeting the EZH2-H3K27me3TET1 pathway, which can modulate the epigenetic landscape. In 2020, Bin Bao et al. [51] demonstrated that TET1- and TET1-dependent $5 \mathrm{hmC}$ mediated a novel hydrogen peroxide- $\left(\mathrm{H}_{2} \mathrm{O}_{2}\right)$-dependent gene expression cascade response driving the self-renewal and expansion of cancer stem cell-like cells (CSCs) in TNBC.

According to the study of Charly Ryan Good, the expression of TET1 was significantly suppressed in HRBC patients while unchanged in HER2-positive cases. This differential result suggests that the degree of TET1 expression varies among different subtypes of breast cancer. Hence, it is necessary to fully consider different subtypes of breast cancer when exploring therapeutic targets for breast cancer based on the suppressive effect of TET1 on related genes.

\section{TET1 in lung cancer}

The role TET1 plays in lung cancer remains controversial. In 2016 Matteo Forloni et al. [52] demonstrated that EGFR triggered the silencing of multiple tumour suppressors in lung adenocarcinoma via $\mathrm{C} / \mathrm{EBP} \alpha$ transcription factor by inhibiting TET1 expression. After EGFR inhibition, TET1 binds to the promotor of the tumour suppressor genes and induces its repression through DNA demethylation. Ectopic expression of TET1 effectively inhibits the growth of the lung cancer, while knockdown of TET1 causes lung adenocarcinoma cells to develop resistance to EGFR inhibitors. Usually, TET1 in lung cancer showed decreased expression or cytoplasmic localization. In 2017, a study conducted by J-I Lai et al. [53] found that patients with higher TET1 levels showed a trend towards increased response efficiency to EGFR inhibitors compared to patients with lower TET1 expression, but the trend was not significant $(p=0.08)$. Furthermore, no correlation was observed between TET1 expression levels and patient survival. Although the oncogenic role of TET1 inhibition against EGFR has been established in cellular and animal models of lung cancer, its role in patient prognosis is still inconclusive and worth further investigation. 
In 2019 Piotr T Filipczak et al. [54] found overexpression of TET1 in lung adenocarcinoma and squamous cell carcinoma. They demonstrated that knockdown of TET1 inhibited cell growth in vitro and in vivo and induced transcriptome reprogramming, affecting critical signalling pathways in cancer, but this effect was independent of its demethylation activity. Wild-type p53 binds to the promoter of TET 1 to repress transcription, and p53 mutations are closely associated with high TET1 expression. Knockdown of TET1 in p53 mutant cell lines successfully induced cellular senescence. These data identify TET1 as a proto-oncogene in lung cancer, and its enhanced function following p53 deletion may be exploited by targeted therapy-induced senescence. In 2021 Hong-Qiang Chen et al. [55] found that TET1 expression was significantly downregulated in 3methylcholanthrene (3-MCA)-induced cellular malignant transformation model, rat chemical carcinogenesis model and human lung cancer tissues. Both in vitro and in vivo experiments showed that TET1 overexpression inhibited lung cancer cell proliferation, migration and invasion, while knockdown of TET1 resulted in the opposite effect. In malignantly transformed cells, the promoters of XRCC1, human 8-ox-oguanine DNA glycosylase 1 (OGG1), and DNA-(apurinic or apyrimidinic site) endonuclease (APEX1), key genes of the BER pathway, showed significantly lower levels of DNA hydroxymethylation and significantly higher levels of methylation. After differential expression of TET1, the hydroxymethylation, methylation and expression levels of these genes were also significantly altered. In addition, TET1 binds to XRCC1, OGG1 and APEX1 to maintain their hydroxymethylation, and blocking one or several essential genes in the BER pathway significantly inhibits the effect of TET1. This study demonstrates for the first time that TET1 expression is regulated by DNA methylation during 3-MCA-induced lung carcinogenesis and that TET1-mediated hydroxymethylation regulates the BER pathway and inhibits cancer cell proliferation, migration and invasion.

\section{TET1 in hepatocellular carcinoma and cholangiocarcinoma}

The role of TET1 in hepatocellular carcinoma has not been thoroughly investigated. Available studies have confirmed that TET1 expression is suppressed in hepatocellular carcinoma (HCC), cells cultured in vitro, and some rapidly proliferating hepatocytes after hepatectomy [56], yet there is no significant change in TET1 transcript levels [56, 57].

The progression of cholangiocarcinoma (CCA) was highly correlated with TET1. Knockdown of TET1 can epigenetically inhibit CCA progression through targeted regulation of cell growth and apoptosis. In a liver in situ xenograft model, suppressing TET1 expression remarkably inhibited CCA malignant progression by inhibiting cell growth and inducing apoptosis [58]. TET1 decreases in intrahepatic cholangiocarcinoma (ICC) and suppresses ICC progression by activating oncogenes through direct binding to oncogene promoters for demethylation [59].

\section{TET1 in colon cancer}

DNA methylation abnormalities are common in colon cancer (CC). Current studies suggest that TET1 acts as a suppressor of CC through the Wnt signalling pathway. In 2015, F Neri et al. [60] considered TET1 as a tumour suppressor that inhibits colon cancer growth by antagonizing inhibitors of the Wnt signalling pathway. TET1 binds to the promoter of the inhibitor of the DKK gene, one of the components of the Wnt signalling pathway, to maintain its hypomethylation. In 2019, Hailong Guo et al. [61] demonstrated that TET1 inhibited CC proliferation by suppressing the $\beta$-catenin signalling pathway.

\section{TET1 in pancreatic cancer}

Similar to CC, TET1 inhibits pancreatic cancer progression by blocking the Wnt signalling pathway and suppresses pancreatic tumour proliferation and metastasis in vivo and in vitro. TET1 binds to the secreted frizzledrelated protein 2 (SFRP2) promoter and catalyses its demethylation to activate SFRP2 transcription, thereby repressing the $\mathrm{Wnt} / \beta$-catenin signalling pathway and $\mathrm{ul}$ timately restraining the epithelial-mesenchymal transition (EMT) in pancreatic tumours. Pancreatic cancer patients with low TET1 expression levels have shorter survival rates than those with higher TET1 levels [62].

TET1 suppresses the Hedgehog signalling pathway through demethylation of CHL1 to downregulate EMT and chemoresistance in pancreatic ductal adenocarcinoma [63].

\section{TET1 in prostate cancer}

TET1 is downregulated in prostate cancer tissues. In xenograft models, TET1 depletion promotes tumour cell growth, invasion and metastasis [64]. TET1 inhibits prostate cancer invasion by activating tissue inhibitors of metalloproteinases [44].

\section{TET1 in cervical cancer}

In 2019, Po-Hsuan Su et al. [65] found increased TET1 and $5 \mathrm{hmC}$ correlation from normal to low-grade squamous intraepithelial lesions (LSIL) in the cervix, maximized in high-grade squamous intraepithelial lesions (HSIL), and reduced in invasive carcinoma. Full-length HPV-immortalized HSIL cells demonstrated higher TET1/5hmC levels and stemness properties than invasive cancer cells. TET1 silencing promoted EMT to transform precancerous cells in vivo. TET1 increased 
$5 \mathrm{hmC}$ in the zinc finger E-box binding homeobox 1 (ZEB1) and Vimentin (VIM) promoters; surprisingly, both genes were silenced. TET1 interaction with the histone modifiers lysine specific demethylase 1 (LSD1) and EZH2 on the ZEB1 promoter resulted in gene silencing via loss of tri-methylation at histone lysine 4 of histone (H3K4me3) and gain of histone H3K27me3. Taken together, TET1 promotes stemness properties and inhibits EMT in HSIL cells through $5 \mathrm{hmC}$-dependent and $5 \mathrm{hmC}$-independent mechanisms. Recently, several studies have proposed that TET1 interacts with the immune system to influence the epigenetics of cancer cells. In 2019, Hao-Xiang Wu et al. [37] collected and consolidated clinical cohorts with annotated response and survival data and matched mutational data from published studies. The predictive function of specific mutated genes was first tested in the discovery cohort and later validated in the validation cohort. The association between specific mutated genes and tumour immunogenicity and antitumour immunity was further investigated in TCGA dataset. TET1 was recurrently mutated across multiple cancers and more frequently seen in skin, lung, gastrointestinal, and urogenital cancers. Collignon et al. [45] demonstrated in their study of basal-like breast cancer (BLBC) that TET1 inhibition was associated with high expression of immune markers and high infiltration of immune cells. In BLBC tissues, there was a negative correlation between TET1 expression and nuclear factor $\kappa B(N F-\kappa B)$, the main immune regulator family.

\section{TET1 in metabolism}

TET1 is an autonomous blocker of critical thermogenic genes (including UCP1 and PPARGC1a) in brown adipocytes. TET1 knockout mice showed improved cold tolerance, increased energy expenditure, and suppression of diet-induced obesity and insulin resistance. Furthermore, the repressive role of TET1 in thermogenic gene regulation in brown adipocytes is largely independent of DNA demethylase but coordinates with the proto-oncogene histone deacetylase 1 (HDAC1) to mediate epigenetic changes to repress thermogenic gene transcription [66].

Nonalcoholic fatty liver disease (NAFLD) is a lipid metabolism disease and an important driver of cirrhosis and hepatocellular carcinoma. TET1 promotes fatty acid oxidation and inhibits NAFLD progression through hydroxymethylation of the peroxisome proliferatoractivated receptors $\alpha($ PPAR $\alpha)$ promoter $[67,68]$.

TET1 stabilizes hypoxia-inducible factor $\alpha$ (HIF- $\alpha)$ and enhances HIF- $\alpha$ transcription activity independent of its enzymatic activity. TET1 competes with prolyl hydroxylase protein 2 (PHD2) to bind to HIF-2 $\alpha$, resulting in a reduction in HIF-2 $\alpha$ hydroxylation by PHD2. However, TET1 has no effect on HIF-1 $\alpha$ hydroxylation, but rather it appears to stabilize the C-terminus of HIF- $1 \alpha$ by affecting lysine site modification.

\section{TET1 in the neurological system}

TET1 is closely associated with psychiatric disorders (e.g., schizophrenia, autism). Upregulation of TET1 expression in the genic parietal cortex of patients with psychiatric disorders increases the level of $5 \mathrm{hmC}$ in promoter region 67 of brain glutamic acid decarboxylase, induces activation of AID/APOBEC expression, and downregulates and impairs the $5 \mathrm{hmU}$ base excision repair pathway. These findings may play a role in the pathophysiological mechanisms of certain psychiatric disorders [69]. TET1 isoforms differentially regulate gene expression, synaptic transmission and memory in the mammalian brain [70,71].

Life experiences can leave lasting marks in the brain, such as epigenetic changes. How life experiences are translated into storable epigenetic information remains largely unknown. Current studies have demonstrated that early growth response 1 (EGR1) recruits TET1 to remove methylation marks and activate downstream genes, providing new insight into how life experiences may shape the brain methylome [72].

TET1 has recently been suggested to be linked to pain hypersensitivity, TET1 demethylates the mGluR5 promoter to cause pain, and this effect can be alleviated by melatonin [73].

\section{Conclusions}

DNA demethylation is an important aspect of epigenetic modification and TET1 plays an important role in this process. Although the exact mechanism has yielded promising results with advanced studies, some details have still not been clarified. For example, the direct decarboxylation of $5 \mathrm{caC}$ decarboxylase in vivo has not been determined, and the interaction between TET1 and DNMT in the process of DNMT-mediated decarboxylation still deserves further exploration. In addition, the role of TET1 in tumours remains controversial. The loss of function of $\mathrm{CpG}$ islands in the promoter due to abnormal hypermethylation is an important pathway for antioncogene inactivation. In most tumours, TET1 reverses the inactivation of hypermethylated antioncogenes and acts as an "activator". Recent studies suggest that TET1 is differentially expressed in different types of tumours and different subtypes of the same tumour and exhibits different biological effects by activating different genes in different signalling pathways, thus displaying both oncogene and antioncogene properties. However, the exact mechanism needs to be further clarified. The research of regarding TET1 as a potential therapeutic target has also made some progress, especially in T-ALL, TNBC and lung adenocarcinoma. But its complex role 
in different signal pathways has limited its application. TET1 has been studied in stem cells, neuroregeneration, metabolism, ageing, and psychiatric diseases but not yet in depth. In recent years, TET1 has also attracted attention due to its noncatalytic role in early neuronal differentiation. In conclusion, research on the TET1 protein will provide a wider range of ideas for epigenetic studies, mechanisms of related diseases and new therapeutic targets. Research on the TET1 protein has broad prospects and is of great value in medicine.

\section{Abbreviations}

TET: Ten-eleven translocation; 5mC: 5-methylcytosine; DNMT: DNA methyltransferase; SAM: S-adenosyl methionine; 5C: 5-cytosine; CD: Cysteinerich domain; CXXC: Cys-Xaa-Xaa-Cys; DSBH: Double-stranded b-helix; 5hmC: 5-hydroxymethylcytosine; UHRF1: Ubiquitin-like with PHD and ring finger domains 1; BER: Base excision repair; TDG: Thymidine-DNA glycosylase; 5fC: 5-Formylcytosine; 5caC: 5-carboxylcytosine; XRCC1: X-ray repair cross complementary protein 1; LIG3: DNA ligase 3; POLb: DNA polymerase b; AID: Activation-induced deaminase; APOBEC: Apolipo protein B mRNAediting enzyme-catalyzed polypeptide-like; 5hmU: 5-hydroxymethyluracil; AP: Apurinic/apyrimidinic; APE1/Ref-1: Apurinic aprimidinic endonuclease/ Redox factor-1; GADD45: Growth arrest and DNA damage inducible 45; MBD4: Growth arrest and DNA damage inducible 45; dsDNA: Doublestranded DNA; ESCs: Embryonic stem cells; ES: Embryonic stem; PRC2: Polycomb repressive complex 2; H3K27me3: Tri-methylation at lysine 27 of histone H3; EED: Embryonic ectoderm development; EZH2: Enhancer of zeste 2; SUZ12: Suppressor of zeste 12; JMJD8: Jumonji domain-containing protein 8; PRC1: Polycomb repressive complex 1; BMMSCs: Bone marrow mesenchymal stem cells; P2RX7: P2X purinoceptor 7; TCGA: The Cancer Genome Atlas; ORR: Objective response rate; DCB: Durable clinical benefit; PFS: Progression-free survival; OS: Overall survival; Treg: Regulator T; FOXP3: Forkhead box P3; AML: Acute myeloid leukemia; MLL: Mixed lineage leukemia; T-ALL: T-cell acute lymphoblastic leukemia; MDS: Myelodysplastic syndromes; CML: Chronic myelocytic leukemia; FECR1: FLI1 exonic circular RNA; TNBC: Triple-negative breast cancer; HER2: Human epidermal growth factor receptor 2; HRBC: Hormone receptor positive;

PI3K: Phosphatidylinositol 3-kinase; EGFR: Epidermal growth factor receptor; PDGF: Platelet derived growth factor; CSCs: Cancer stem cell-like cells; 3MCA: 3-methylcholanthrene; OGG1: Human 8-ox-oguanine DNA glycosylase 1; APEX1: DNA- (apurinic or apyrimidinic site) endonuclease;

HCC: Hepatocellular carcinoma; CCA: Cholangiocarcinoma; ICC: Intrahepatic cholangiocarcinoma; CC: Colon cancer; SFRP2: Secreted frizzled-related protein 2; EMT: Epithelial-mesenchymal transition; LSIL: Low-grade squamous intraepithelial lesions; HSIL: High-grade squamous intraepithelial lesions; ZEB1: Zinc finger E-box binding homeobox 1; VIM: Vimentin; LSD1: Lysine specific demethylase 1; H3K4me3: Tri-methylation at lysine 4 of histone H3; BLBC: Basal-like breast cancer; NF-kB: Nuclear factor kB; HDAC1: Histone deacetylase 1; NAFLD: Non-alcoholic fatty liver disease; PPARa: Peroxisome proliferator-activated receptors a; HIF-a: Hypoxia-inducible factor a; PHD2: Prolyl hydroxylase protein 2; EGR1: Early growth response 1

\section{Acknowledgements}

Not applicable.

\section{Authors' contributions}

Wenzheng Liu wrote the initial manuscript. Guanhua Wu, Fei Xiong and Yongjun Chen revised the manuscript. All authors read and approved the final manuscript.

\section{Funding}

This study was funded by National Natural Science Foundation (No 81572783, No. 81974438).

Availability of data and materials Not applicable.

\section{Declarations}

Ethics approval and consent to participate

Not applicable.

\section{Consent for publication}

Not applicable.

\section{Competing interests}

The authors declare that they have no competing interests.

Received: 26 July 2021 Accepted: 3 October 2021

Published online: 16 October 2021

\section{References}

1. Ono R, Taki T, Taketani T, Taniwaki M, Kobayashi H, Hayashi Y. LCX, leukemia-associated protein with a CXXC domain, is fused to MLL in acute myeloid leukemia with trilineage dysplasia having t (10;11)(q22;q23). Cancer Res. 2002;62(14):4075-80. Published July 2002.

2. Tahiliani M, Koh KP, Shen Y, Pastor WA, Bandukwala H, Brudno Y, et al. Conversion of 5-methylcytosine to 5-hydroxymethylcytosine in mammalian DNA by MLL partner TET1. Science. 2009;324(5929):930-5. https://doi.org/1 $0.1126 /$ science. 1170116 .

3. Ito S, D'Alessio AC, Taranova OV, Hong K, Sowers LC, Zhang Y. Role of Tet proteins in $5 \mathrm{mC}$ to $5 \mathrm{hmC}$ conversion, ES-cell self-renewal and inner cell mass specification. Nature. 2010;466(7310):1129-33. https://doi.org/10.1038/ nature09303.

4. Koh KP, Yabuuchi A, Rao S, Huang Y, Cunniff K, Nardone J, et al. Tet1 and Tet2 regulate 5 -hydroxymethylcytosine production and cell lineage specification in mouse embryonic stem cells. Cell Stem Cell. 2011;8(2):20013. https://doi.org/10.1016/.j.stem.2011.01.008.

5. Iyer LM, Tahiliani M, Rao A, Aravind L. Prediction of novel families of enzymes involved in oxidative and other complex modifications of bases in nucleic acids. Cell Cycle. 2009;8(11):1698-710. https://doi.org/10.4161/cc.8.11 8580

6. Ito S, Shen L, Dai Q, Wu SC, Collins LB, Swenberg JA, et al. Tet proteins can convert 5-methylcytosine to 5-formylcytosine and 5-carboxylcytosine. Science. 2011;333(6047):1300-3. https://doi.org/10.1126/science.1210597.

7. He Y-F, Li B-Z, Li Z, Liu P, Wang Y, Tang Q, et al. Tet-mediated formation of 5-carboxylcytosine and its excision by TDG in mammalian DNA. Science. 2011;333(6047):1303-7. https://doi.org/10.1126/science.1210944.

8. Goll MG, Bestor TH. Eukaryotic cytosine methyltransferases. Annu Rev Biochem. 2005;74(1):481-514. https://doi.org/10.1146/annurev.biochem.74.01 0904.153721.

9. Bogdanović $\mathrm{O}$, Smits $\mathrm{AH}$, de la Calle ME, Tena JJ, Ford E, Williams R, et al. Active DNA demethylation at enhancers during the vertebrate phylotypic period. Nat Genet. 2016;48(4):417-26. https://doi.org/10.1038/ng.3522.

10. Bostick M, Kim JK, Estève P-O, Clark A, Pradhan S, Jacobsen SE. UHRF1 plays a role in maintaining DNA methylation in mammalian cells. Science. 2007;317(5845):1760-4. https://doi.org/10.1126/science.1147939. Epub 2007 Aug 2.

11. Hashimoto H, Liu Y, Upadhyay AK, Chang Y, Howerton SB, Vertino PM, et al. Recognition and potential mechanisms for replication and erasure of cytosine hydroxymethylation. Nucleic Acids Res. 2012;40(11):4841-9. https:// doi.org/10.1093/nar/gks155.

12. $\mathrm{Xu}$ C, Corces VG. Nascent DNA methylome mapping reveals inheritance of hemimethylation at CTCF/cohesin sites. Science. 2018;359(6380):1166-70. https://doi.org/10.1126/science.aan5480.

13. Kubosaki A, Tomaru Y, Furuhata E, Suzuki T, Shin JW, Simon C, et al. CpG site-specific alteration of hydroxymethylcytosine to methylcytosine beyond DNA replication. Biochem Biophys Res Commun. 2012;426(1):141-7. https:// doi.org/10.1016/j.bbrc.2012.08.053.

14. Cortellino S, Xu J, Sannai M, Moore R, Caretti E, Cigliano A, et al. Thymine DNA glycosylase is essential for active DNA demethylation by linked deamination-base excision repair. Cell. 2011;146(1):67-79. https://doi.org/1 0.1016/j.cell.2011.06.020.

15. Cortázar D, Kunz C, Saito $Y$, Steinacher R, Schär P. The enigmatic thymine DNA glycosylase. DNA Repair (Amst). 2007;6(4):489-504. https://doi.org/10.1 016/j.dnarep.2006.10.013. Epub 2006 Nov 20.

16. Weber AR, Krawczyk C, Robertson AB, Kuśnierczyk A, Vågbø CB, Schuermann $D$, et al. Biochemical reconstitution of TET1-TDG-BER-dependent active DNA 
demethylation reveals a highly coordinated mechanism. Nat Commun. 2016;7(1). https://doi.org/10.1038/ncomms10806.

17. Guo JU, Su Y, Zhong C, G-I M, Song H. Hydroxylation of 5-methylcytosine by TET1 promotes active DNA demethylation in the adult brain. Cell. 2011 https://doi.org/10.1016/j.cell.2011.03.022.

18. Bird $\mathrm{A}$. The dinucleotide CG as a genomic signalling module. J Mol Biol. 2011;409(1):47-53. https://doi.org/10.1016/j.jmb.2011.01.056.

19. Niehrs C, Schäfer A. Active DNA demethylation by Gadd45 and DNA repair. Trends Cell Biol. 2012;22(4):220-7. https://doi.org/10.1016/j.tcb.2012.01.002.

20. Rai K, Huggins IJ, James SR, Karpf AR, Jones DA, Cairns BR. DNA demethylation in zebrafish involves the coupling of a deaminase, a glycosylase, and gadd45. Cell. 2008;135(7):1201-12. https://doi.org/10.1016/j. cell.2008.11.042.

21. Barreto G, Schäfer A, Marhold J, Stach D, Swaminathan SK, Handa V, et al. Gadd45a promotes epigenetic gene activation by repair-mediated DNA demethylation. Nature. 2007;445(7128):671-5. https://doi.org/10.1038/na ture05515.

22. Bhutani N, Brady JJ, Damian M, Sacco A, Corbel SY, Blau HM. Reprogramming towards pluripotency requires AID-dependent DNA demethylation. Nature. 2010;463(7284):1042-7. https://doi.org/10.1038/nature08752.

23. Popp C, Dean W, Feng S, Cokus SJ, Andrews S, Pellegrini M, et al. Genomewide erasure of DNA methylation in mouse primordial germ cells is affected by AID deficiency. Nature. 2010;463(7284):1101-5. https://doi.org/10.1038/na ture08829.

24. Bransteitter R, Pham P, Scharff MD, Goodman MF. Activation-induced cytidine deaminase deaminates deoxycytidine on single-stranded DNA but requires the action of RNase. Proc Natl Acad Sci U S A. 2003;100(7):4102-7. https://doi.org/10.1073/pnas.0730835100.

25. Nabel CS, Jia H, Ye Y, Shen L, Goldschmidt HL, Stivers JT, et al. AID/APOBEC deaminases disfavor modified cytosines implicated in DNA demethylation. Nat Chem Biol. 2012;8(9):751-8. https://doi.org/10.1038/nchembio.1042.

26. Schiesser S, Hackner B, Pfaffeneder T, Müller M, Hagemeier C, Truss M, et al. Mechanism and stem-cell activity of 5-carboxycytosine decarboxylation determined by isotope tracing. Angew Chem Int Ed Engl. 2012;51(26):651620. https://doi.org/10.1002/anie.201202583.

27. Liutkevičiūtè Z, Kriukienè E, Ličytè J, Rudytè M, Urbanavičiūtè G, Klimašauskas S. Direct decarboxylation of 5-carboxylcytosine by DNA C5methyltransferases. J Am Chem Soc. 2014;136(16):5884-7. https://doi.org/1 $0.1021 / j a 5019223$.

28. Pulecio J, Verma N, Mejía-Ramírez E, Huangfu D, Raya A. CRISPR/Cas9-based engineering of the epigenome. Cell Stem Cell. 2017;21(4):431-47. https:// doi.org/10.1016/j.stem.2017.09.006.

29. Wu H, D'Alessio AC, Ito S, Xia K, Wang Z, Cui K, et al. Dual functions of Tet in transcriptional regulation in mouse embryonic stem cells. Nature. 2011; 473(7347):389-93. https://doi.org/10.1038/nature09934.

30. Gu T, Lin X, Cullen SM, Luo M, Jeong M, Estecio M, et al. DNMT3A and TET1 cooperate to regulate promoter epigenetic landscapes in mouse embryonic stem cells. Genome Biol. 2018;19(1):88. https://doi.org/10.1186/s13059-01 8-1464-7.

31. Verma N, Pan H, Doré LC, Shukla A, Li QV, Pelham-Webb B, et al. TET proteins safeguard bivalent promoters from de novo methylation in human embryonic stem cells. Nat Genet. 2018;50(1):83-95. https://doi.org/10.1038/ s41588-017-0002-y.

32. Senner CE, Chrysanthou S, Burge S, Lin H-Y, Branco MR, Hemberger M. TET1 and 5 -Hydroxymethylation preserve the stem cell state of mouse trophoblast. Stem Cell Reports. 2020;15(6):1301-16. https://doi.org/10.1016/j. stemcr.2020.04.009.

33. Li W, Karwacki-Neisius V, Ma C, Tan L, Shi Y, Wu F, et al. Nono deficiency compromises TET1 chromatin association and impedes neuronal differentiation of mouse embryonic stem cells. Nucleic Acids Res. 2020;48(9): 4827-38. https://doi.org/10.1093/nar/gkaa213.

34. Khoueiry R, Sohni A, Thienpont B, Luo X, Velde JV, Bartoccetti M, et al. Lineage-specific functions of TET1 in the postimplantation mouse embryo. Nat Genet. 2017:49(7):1061-72. https://doi.org/10.1038/ng.3868.

35. Hill PWS, Leitch HG, Requena CE, Sun Z, Amouroux R, Roman-Trufero M, et al. Epigenetic reprogramming enables the transition from primordial germ cell to gonocyte. Nature. 2018;555(7696):392-6. https://doi.org/10.103 8/nature25964.

36. Yang R, Yu T, Kou X, Gao X, Chen C, Liu D, et al. Tet1 and Tet2 maintain mesenchymal stem cell homeostasis via demethylation of the $\mathrm{P} 2 \mathrm{rX} 7$ promoter. Nat Commun. 2018;9(1):2143. https://doi.org/10.1038/s41467-01804464-6.

37. Wu H-X, Chen Y-X, Wang Z-X, Zhao Q, He M-M, Wang Y-N, et al. Alteration in TET1 as potential biomarker for immune checkpoint blockade in multiple cancers. J Immunother Cancer. 2019;7(1):264. https://doi.org/10.1186/s4042 5-019-0737-3.

38. Yang R, Qu C, Zhou Y, Konkel JE, Shi S, Liu Y, et al. Hydrogen sulfide promotes Tet1- and Tet2-mediated Foxp3 demethylation to drive regulatory T cell differentiation and maintain immune homeostasis. Immunity. 2015; 43(2):251-63. https://doi.org/10.1016/j.immuni.2015.07.017.

39. Huang $H$, Jiang $X$, Li Z, Li Y, Song C-X, He C, et al. TET1 plays an essential oncogenic role in MLL-rearranged leukemia. Proc Natl Acad Sci U S A. 2013; 110(29):11994-9. https://doi.org/10.1073/pnas.1310656110.

40. Zhang T, Zhao Y, Zhao Y, Zhou J. Expression and prognosis analysis of family in acute myeloid leukemia. Aging (Albany NY). 2020. https://doi.org/1 0.18632 /aging. 102928

41. Bamezai S, Demir D, Pulikkottil AJ, Ciccarone F, Fischbein E, Sinha A, et al. TET1 promotes growth of T-cell acute lymphoblastic leukemia and can be antagonized via PARP inhibition. Leukemia. 2021;35(2):389-403. https://doi. org/10.1038/s41375-020-0864-3.

42. Abdel-Wahab O, Mullally A, Hedvat C, Garcia-Manero G, Patel J, Wadleigh M, et al. Genetic characterization of TET1, TET2, and TET3 alterations in myeloid malignancies. Blood. 2009;114(1):144-7. https://doi.org/10.1182/blood-2009-03-21 0039.

43. Solary E, Bernard OA, Tefferi A, Fuks F, Vainchenker W. The ten-eleven Translocation-2 (TET2) gene in hematopoiesis and hematopoietic diseases. Leukemia. 2014;28(3):485-96. https://doi.org/10.1038/leu.2013.337.

44. Hsu C-H, Peng K-L, Kang M-L, Chen Y-R, Yang Y-C, Tsai C-H, et al. TET1 suppresses cancer invasion by activating the tissue inhibitors of metalloproteinases. Cell Rep. 2012;2(3):568-79. https://doi.org/10.1016/j. celrep.2012.08.030

45. Collignon E, Canale A, AI Wardi C, Bizet M, Calonne E, Dedeurwaerder S, et al. Immunity drives regulation in cancer through NF-KB. Sci Adv. 2018, 4(6):eaap7309. https://doi.org/10.1126/sciadv.aap7309.

46. Haffner MC, Chaux A, Meeker AK, Esopi DM, Gerber J, Pellakuru LG, et al. Global 5-hydroxymethylcytosine content is significantly reduced in tissue stem/progenitor cell compartments and in human cancers. Oncotarget. 2011. https://doi.org/10.18632/oncotarget.316.

47. Lian CG, Xu Y, Ceol C, Wu F, Larson A, Dresser K, et al. Loss of 5hydroxymethylcytosine is an epigenetic hallmark of melanoma. Cell. 2012; 150(6):1135-46. https://doi.org/10.1016/j.cell.2012.07.033.

48. Chen N, Zhao G, Yan X, Lv Z, Yin H, Zhang S, et al. A novel FLI1 exonic circular RNA promotes metastasis in breast cancer by coordinately regulating TET1 and DNMT1. Genome Biol. 2018;19(1):218. https://doi.org/1 0.1186/s13059-018-1594-y.

49. Good CR, Panjarian S, Kelly AD, Madzo J, Patel B, Jelinek J, et al. TET1mediated Hypomethylation activates oncogenic signaling in triple-negative breast Cancer. Cancer Res. 2018;78(15):4126-37. https://doi.org/10.1158/ 0008-5472.CAN-17-2082.

50. Yu Y, Qi J, Xiong J, Jiang L, Cui D, He J, et al. Epigenetic co-deregulation of EZH2/TET1 is a senescence-countering, actionable vulnerability in triplenegative breast Cancer. Theranostics. 2019;9(3):761-77. https://doi.org/10.71 50/thno.29520.

51. Bao B, Teslow EA, Mitrea C, Boerner JL, Dyson G, Bollig-Fischer A. Role of TET1 and 5hmC in an obesity-linked pathway driving Cancer stem cells in triple-negative breast Cancer. Mol Cancer Res. 2020;18(12):1803-14. https:// doi.org/10.1158/1541-7786.MCR-20-0359.

52. Forloni M, Gupta R, Nagarajan A, Sun L-S, Dong Y, Pirazzoli V, et al. Oncogenic EGFR represses the TET1 DNA demethylase to induce silencing of tumor suppressors in Cancer cells. Cell Rep. 2016;16(2):457-71. https://doi. org/10.1016/j.celrep.2016.05.087.

53. Lai Jl, Lai YC, Chen YC, Wang NK, Pan JN, Wang WS, et al. Clinical analysis of NSCLC patients reveals lack of association between EGFR mutation and TET1 downregulation. Cancer Gene Ther. 2017;24(9):373-80. https://doi. org/10.1038/cgt.2017.26

54. Filipczak PT, Leng S, Tellez CS, Do KC, Grimes MJ, Thomas CL, et al. p53suppressed oncogene TET1 prevents cellular aging in lung Cancer. Cancer Res. 2019:79(8):1758-68. https://doi.org/10.1158/0008-5472.CAN-18-1234.

55. Chen H-Q, Chen D-J, Li Y, Yuan W-B, Fan J, Zhang Z, et al. Epigenetic silencing of TET1 mediated hydroxymethylation of base excision repair 
pathway during lung carcinogenesis. Environ Pollut. 2021;268(Pt B):115860 https://doi.org/10.1016/j.envpol.2020.115860.

56. Thomson JP, Ottaviano R, Unterberger EB, Lempiäinen $H$, Muller $A$, Terranova $R$, et al. Loss of Tet1-associated 5-Hydroxymethylcytosine is concomitant with aberrant promoter Hypermethylation in liver Cancer. Cancer Res. 2016;76(10):3097-108. https://doi.org/10.1158/0008-5472.CAN-1 5-1910.

57. Koike H, Ouchi R, Ueno Y, Nakata S, Obana Y, Sekine K, et al. Polycomb group protein Ezh2 regulates hepatic progenitor cell proliferation and differentiation in murine embryonic liver. PLoS One. 2014;9(8):e104776. https://doi.org/10.1371/journal.pone.0104776.

58. Bai X, Zhang H, Zhou Y, Nagaoka K, Meng J, Ji C, et al. TET1 promotes malignant progression of cholangiocarcinoma with IDH1 wild-type. Hepatology. 2020;73(5):1747-63. https://doi.org/10.1002/hep.31486.

59. Li H, Zhou Z-Q, Yang Z-R, Tong D-N, Guan J, Shi B-J, et al. MicroRNA-191 acts as a tumor promoter by modulating the TET1-p53 pathway in intrahepatic cholangiocarcinoma. Hepatology. 2017;66(1):136-51. https://doi. org/10.1002/hep.29116.

60. Neri F, Dettori D, Incarnato D, Krepelova A, Rapelli S, Maldotti M, et al. TET1 is a tumour suppressor that inhibits colon cancer growth by derepressing inhibitors of the WNT pathway. Oncogene. 2015;34(32):4168-76. https://doi. org/10.1038/onc.2014.356.

61. Guo H, Zhu H, Zhang J, Wan B, Shen Z. TET1 suppresses colon cancer proliferation by impairing $\beta$-catenin signal pathway. J Cell Biochem. 2019; 120(8):12559-65. https://doi.org/10.1002/jcb.28522.

62. Wu J, Li H, Shi M, Zhu Y, Ma Y, Zhong Y, et al. TET1-mediated DNA hydroxymethylation activates inhibitors of the Wnt/ $\beta$-catenin signaling pathway to suppress EMT in pancreatic tumor cells. J Exp Clin Cancer Res. 2019;38(1):348. https://doi.org/10.1186/s13046-019-1334-5.

63. Li H, Jiang W, Liu X-N, Yuan L-Y, Li T-J, Li S, et al. TET1 downregulates epithelial-mesenchymal transition and chemoresistance in PDAC by demethylating $\mathrm{CHL} 1$ to inhibit the hedgehog signaling pathway. Oncogene. 2020;39(36):5825-38. https://doi.org/10.1038/s41388-020-014078 .

64. Brothman AR, Swanson G, Maxwell TM, Cui J, Murphy KJ, Herrick J, et al. Global hypomethylation is common in prostate cancer cells: a quantitative predictor for clinical outcome? Cancer Genet Cytogenet. 2005;156(1):31-6. https://doi.org/10.1016/j.cancergencyto.2004.04.004.

65. Su P-H, Hsu Y-W, Huang R-L, Chen L-Y, Chao T-K, Liao C-C, et al. TET1 promotes $5 \mathrm{hmC}$-dependent stemness, and inhibits a $5 \mathrm{hmC}$-independent epithelial-mesenchymal transition, in cervical precancerous lesions. Cancer Lett. 2019;450:53-62. https://doi.org/10.1016/j.canlet.2019.01.033.

66. Damal Villivalam S, You D, Kim J, Lim HW, Xiao H, Zushin P-JH, et al. TET1 is a beige adipocyte-selective epigenetic suppressor of thermogenesis. Nat Commun. 2020;1 1(1):4313. https://doi.org/10.1038/s41467-020-18054-y.

67. Wang J, Zhang Y, Zhuo Q, Tseng Y, Wang J, Ma Y, et al. TET1 promotes fatty acid oxidation and inhibits NAFLD progression by hydroxymethylation of PPARa promoter. Nutr Metab (Lond). 2020;17(1):46. https://doi.org/10.1186/ s12986-020-00466-8.

68. Ali MM, Phillips SA, Mahmoud AM. HIF1a/TET1 pathway mediates hypoxiainduced Adipocytokine promoter Hypomethylation in human adipocytes. Cells. 2020;9(1). https://doi.org/10.3390/cells9010134.

69. Dong E, Gavin DP, Chen Y, Davis J. Upregulation of TET1 and downregulation of $A P O B E C 3 A$ and $A P O B E C 3 C$ in the parietal cortex of psychotic patients. Transl Psychiatry. 2012;2(9):e159. https://doi.org/10.1038/ tp.2012.86.

70. Greer CB, Wright J, Weiss JD, Lazarenko RM, Moran SP, Zhu J, et al. Isoforms differentially regulate gene expression, synaptic transmission, and memory in the mammalian brain. J Neurosci. 2021;41(4):578-93. https://doi.org/10.1 523/JNEUROSCI.1821-20.2020.

71. Kaas GA, Zhong C, Eason DE, Ross DL, Vachhani RV, Ming G-L, et al. TET1 controls CNS 5-methylcytosine hydroxylation, active DNA demethylation, gene transcription, and memory formation. Neuron. 2013;79(6):1086-93. https://doi.org/10.1016/j.neuron.2013.08.032.

72. Sun Z, Xu X, He J, Murray A, Sun M-A, Wei X, et al. EGR1 recruits TET1 to shape the brain methylome during development and upon neuronal activity. Nat Commun. 2019;10(1):3892. https://doi.org/10.1038/s41467-01 9-11905-3.

73. Hsieh M-C, Ho Y-C, Lai C-Y, Chou D, Wang H-H, Chen G-D, et al. Melatonin impedes Tet1-dependent mGluR5 promoter demethylation to relieve pain. $J$ Pineal Res. 2017;63(4). https://doi.org/10.1111/jpi.12436.

\section{Publisher's Note}

Springer Nature remains neutral with regard to jurisdictional claims in published maps and institutional affiliations.

\section{Ready to submit your research? Choose BMC and benefit from:}

- fast, convenient online submission

- thorough peer review by experienced researchers in your field

- rapid publication on acceptance

- support for research data, including large and complex data types

- gold Open Access which fosters wider collaboration and increased citations

- maximum visibility for your research: over $100 \mathrm{M}$ website views per year

At BMC, research is always in progress.

Learn more biomedcentral.com/submissions 